\title{
La seringue et la gâchette
}

Les genres au rendez-vous de la violence dans Un flic de Jean-Pierre Melville

The syringe and the trigger. Genders present at the rendez-vous with violence in Jean-Pierre Melville's film Un flic

\section{Thomas Lequeu}

\section{OpenEdition}

\section{Journals}

Édition électronique

URL : http://journals.openedition.org/cel/8447

DOI : $10.4000 /$ cel.8447

ISSN : 2262-208X

Éditeur

École du Louvre

Référence électronique

Thomas Lequeu, "La seringue et la gâchette », Les Cahiers de l'École du Louvre [En ligne], 15 | 2020, mis en ligne le 02 novembre 2020, consulté le 05 novembre 2020. URL : http://journals.openedition.org/ cel/8447 ; DOI : https://doi.org/10.4000/cel.8447

Ce document a été généré automatiquement le 5 novembre 2020.

\section{cc) $($ ) $\Theta$}

Les Cahiers de l'École du Louvre sont mis à disposition selon les termes de la licence Creative Commons Attribution - Pas d'Utilisation Commerciale - Pas de Modification 4.0 International. 


\title{
La seringue et la gâchette
}

\author{
Les genres au rendez-vous de la violence dans Un flic de Jean-Pierre \\ Melville
}

The syringe and the trigger. Genders present at the rendez-vous with violence in Jean-Pierre Melville's film Un flic

\section{Thomas Lequeu}

1 Un flic, sorti le 25 octobre 1972, est le dernier film de Jean-Pierre Melville. Il clôt l'exploration du milieu, entamée par le réalisateur en 1955 avec Bob le flambeur. L'intrigue repose ici sur les trajectoires croisées de deux personnages, d'une part le commissaire Édouard Coleman interprété par Alain Delon et d'autre part Simon, le truand, sous les traits de Richard Crenna, d'autre part. Les deux hommes partagent la même femme, Cathy (Catherine Deneuve), compagne de Simon et maîtresse du flic. L'intrigue policière, où Coleman est lancé sur la piste du gang de Simon suite à un braquage qui a mal tourné, devient le prétexte d'une étude des genres dont le film entend présenter un éventail complet. Cathy, figure de la femme fatale, et le double personnage de Coleman-Simon qui incarne l'image du masculin froid et viril, en représentent les deux pôles. Entre ces extrêmes, Melville s'attache à décliner les identités sexuelles dans une galerie de rôles secondaires qui en révèlent un spectre complet. Il y a la femme au foyer dévouée, la prostituée assassinée, Gaby, l'indic transsexuelle, le vieil homosexuel et son jeune éphèbe, le gangster brutal à l'orientation trouble, l'homme lâche qui a perdu sa virilité. Cette question sexuelle (et homosexuelle), omniprésente dans l'univers melvillien, dans les écrits du réalisateur comme dans les entretiens qu'il a accordés, trouve dans Un flic son expression la plus transparente. L'évolution de la réception critique des films de Melville reflète la part de plus en plus évidente que prend la question du genre dans son cinéma. S'il s'est luimême souvent exprimé à ce sujet dès les années 1960, en explicitant notamment la dimension homosexuelle de L'Aîné des Ferchaux (1963) ${ }^{1}$, ou en introduisant des amours saphiques dans Deux hommes dans Manhattan (1959) et Léon Morin, prêtre (1961), la critique semble être longtemps restée muette sur ce sujet. On note à peine la mention d'amitiés viriles dans les premiers articles fleuves qui lui sont consacrés, où l'on s'empresse toutefois de préciser que «cette amitié n'est pas principalement 
sentimentale, et nullement équivoque [...] $]^{2}$ » À l'inverse, les critiques publiées à l'automne 1972 au moment de la sortie d'Un flic évoquent souvent l'ambiguïté de la question sexuelle dans le film, principalement au travers des personnages de Cathy et de Gaby. Le rôle interprété par Catherine Deneuve se trouve tantôt décrit comme "l'expression poétique de l'éternel féminin ${ }^{3}$ ", tantôt comme «une femme transparente ${ }^{4}$ ", "une ravissante blonde (Catherine Deneuve, petit rôle mais stupéfiante beauté) $[. . .]^{5}$.» Le personnage transsexuel intrigue davantage et induit des lectures contradictoires du film. Il est soit un «brave bougre, gentil, sympathique, séduisant [qui] est aussi ignoble dans la mesure où il se doit d'être un professionnel consciencieux et efficace ${ }^{6}$ », soit " un détestable travesti, prostituée de luxe $\mathrm{e}^{7}$ ». Il est intéressant de constater que Gaby - à laquelle tous les articles font référence en utilisant le genre masculin - cristallise une vision morale du film, entre mise en scène $d u$ " dégoût ${ }^{8}$ » et échappatoire à « toutes les simplifications quasi racistes ${ }^{9}$ ». Dès lors, il semble évident que la question sexuelle dépasse largement chez Melville la seule fonction narrative pour devenir un des fondements de son cinéma. Lorsque l'on remonte cette piste dans sa filmographie, les archétypes décrits dans Un flic apparaissent d'autant plus comme l'aboutissement de la construction genrée des individualités dans toute son œuvre. Le destin de Cathy permet ainsi de retracer la lignée des traîtresses melviliennes et de nuancer leur relative passivité, alors que l'étude des figures masculines questionne l'ambiguïté d'un virilisme de façade. Le thème du fétichisme rapproche, en outre, le cinéaste français de ses modèles anglosaxons dans une inversion des sexes particulièrement signifiante.

\section{Femmes létales}

\section{Femme de dos}

2 Cathy apparaitt dans le night-club de Simon après une demi-heure de film. Coleman a pris place au piano dans la salle déserte et, alors que la caméra tourne autour de lui, un contrechamp dévoile le dos de la jeune femme dans l'embrasure d'une porte (fig. 1). On voit d'abord sa nuque et son chignon avant qu'elle ne se retourne progressivement et que son visage n'émerge du recoin noir d'où elle sort à reculons. Cette manière d'apparaître dans un geste peu naturel est rare dans le cinéma de Melville. On en trouve cependant un autre exemple dans Deux hommes dans Manhattan (1959) avec l'entrée en scène de Moreau, personnage interprété par Melville lui-même qui, lui aussi, se montre pour la première fois de dos. Le réalisateur s'est alors expliqué sur ce choix dans les Cahiers du cinéma, pointant « qu'on [ne voit pas] Moreau de face, tout de suite, pour que, dès le départ, il soit ambigu ${ }^{10}$ » Le même soupçon pèse sur Cathy dans Un flic dès ce premier plan, puis la suite du film poursuit cette logique en jouant en permanence sur la dissimulation ou le dévoilement de son visage en fonction de sa situation intime et de son positionnement moral: dans le night-club, le lieu par excellence où elle appartient à Simon, ses cheveux se trouvent toujours tirés, alors qu'ils sont lâchés, ébouriffés et masquent souvent ses traits lorsqu'elle agit pour Coleman. 
Fig. 1

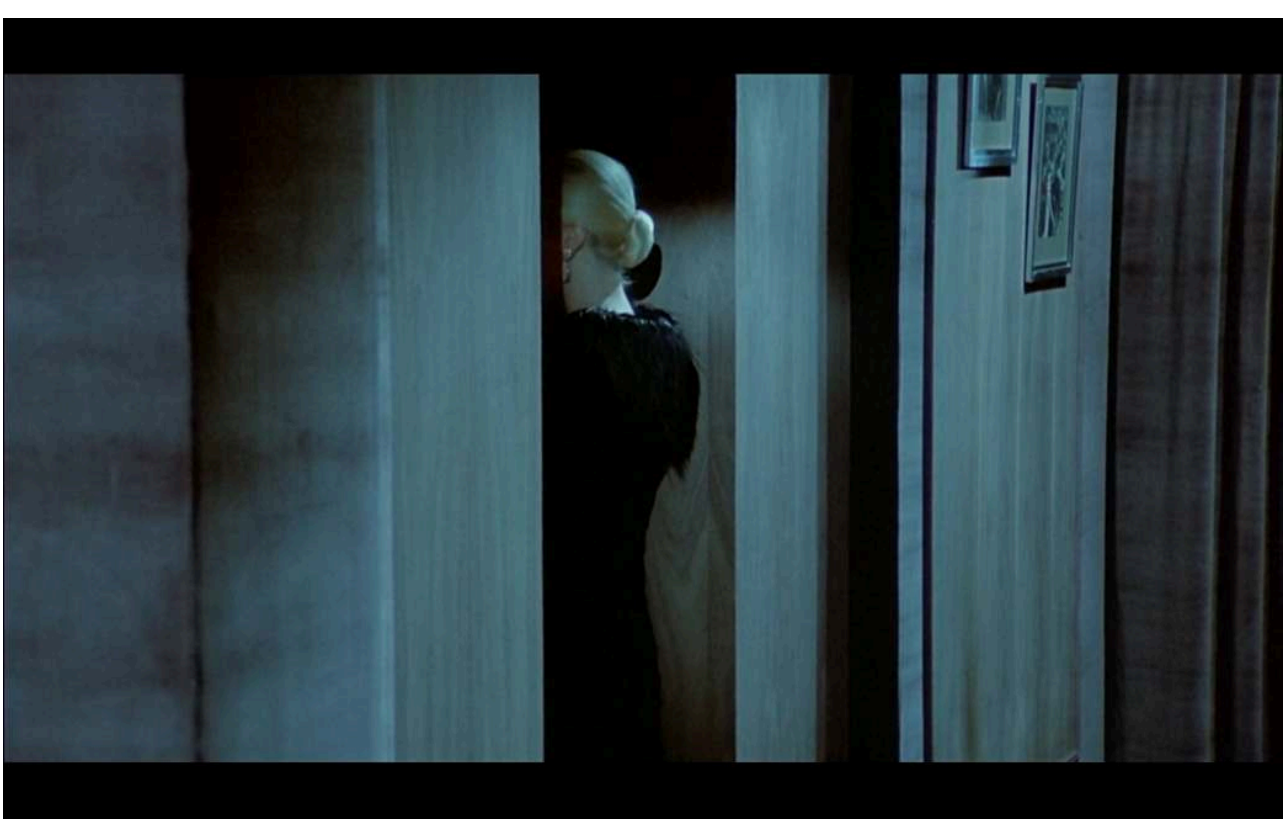

Un flic, Jean-Pierre Melville, 1972

(c) Sophie Dulac Distribution

Ce trouble culmine dans la scène qui entérine la liaison qu'elle entretient avec le commissaire. Elle débute par l'arrivée de Coleman en voiture et se poursuit par son cheminement dans les escaliers et les couloirs d'un hôtel, avant qu'il ne retrouve, dans une chambre, Cathy qui le menace avec son propre revolver. Toute la séquence se déroule comme un jeu de rôle entre les deux amants sous la forme d'un dialogue en faux-semblant dont le spectateur perçoit l'aspect factice par le moyen d'un mouvement d'appareil étrange : un panoramique vertical qui passe du visage de Delon au reflet des deux personnages sur un miroir fixé au plafond (fig. 2). Melville maintient l'ambiguïté qui entoure l'identité de Cathy et la réelle signification de la scène en dissimulant une nouvelle fois les traits de son héroïne dont on ne voit alors que l'épaisse chevelure blonde. Ce plan au miroir revient quelques instants plus tard pour un baiser à double sens, sans leurs deux visages. À bien regarder la scène, elle se trouve entièrement construite comme une variation sur les apparences trompeuses: lorsque Coleman arrive, son image est brouillée, déjà, par les voitures qui passent entre lui et l'objectif. Il met ensuite des lunettes de soleil qui cachent entièrement son regard, puis les miroirs de l'escalier et du couloir reflètent sa silhouette tout au long de son trajet. Nous reviendrons sur l'importance du reflet chez Melville en tant que moyen pour l'homme de se faire face. Au contraire, il semble ici dévoyé ou annulé, les yeux de Coleman restant invisibles derrière des verres fumés devenus eux-mêmes deux petits miroirs dans une mise en abyme qui désincarne le personnage. Cathy, qui représente le centre de gravité de toute la séquence, personnifie un pôle trouble vers lequel on ne peut avancer que masqué aux autres et à soi-même ${ }^{11}$. C'est elle, d'ailleurs, qui prend en charge le sous-texte sexuel menaçant de la scène qui se concentre sur la symbolique violente et phallique du revolver. Elle s'en saisit dans un très léger hors-champ sous la ceinture de Coleman après avoir laissé glisser sa main le long de son buste. Le tenant fermement, elle le dirige contre l'homme dans une prise de pouvoir virile qui déconcerte le commissaire. Enfin, elle le jette négligemment sur l'oreiller où ils feront 
l'amour et où il reste ostensiblement dressé pendant le baiser que nous avons décrit plus haut. Ce geste la désigne comme l'amante dangereuse.

Fig. 2

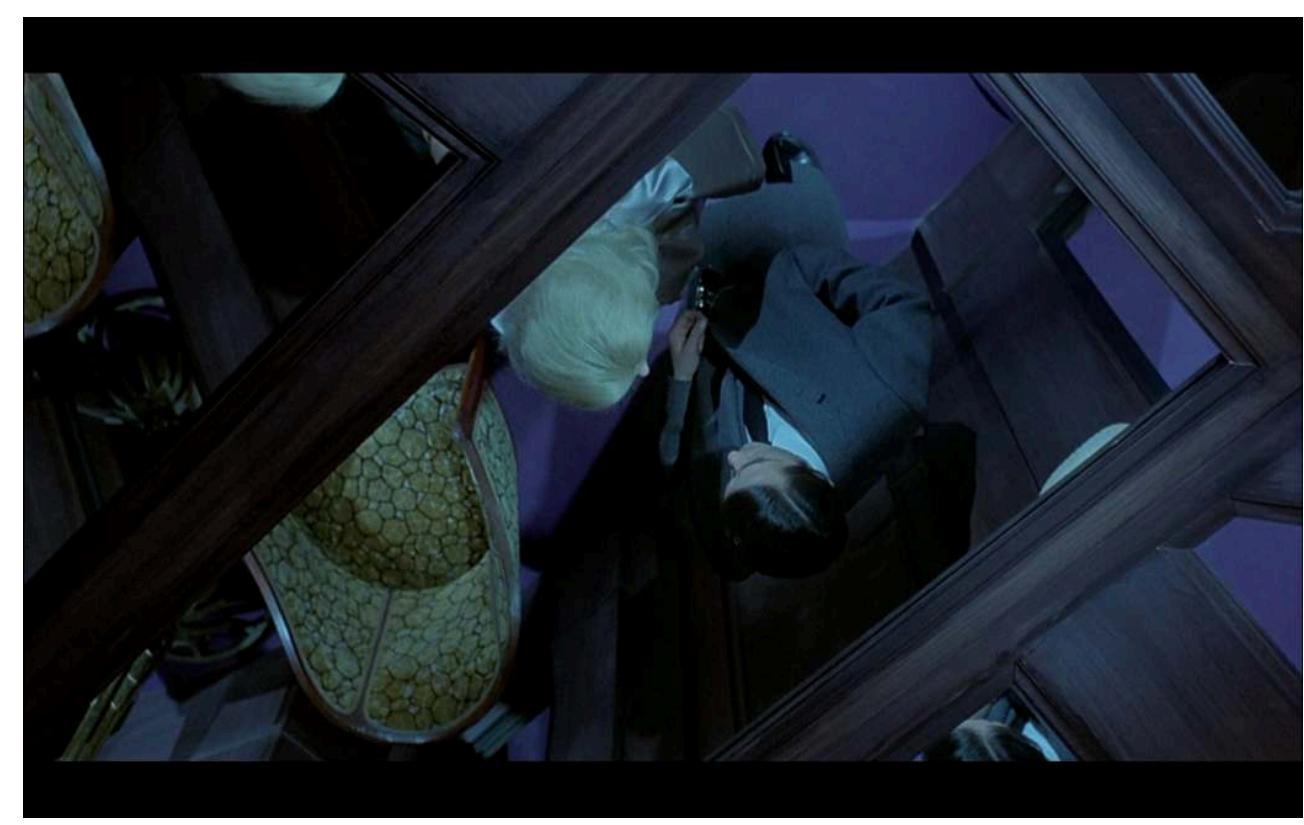

Un flic, Jean-Pierre Melville, 1972

(c) Sophie Dulac Distribution

\section{Le silence des traîtresses}

Le personnage de Cathy incarne à bien des égards l'aboutissement de la figure de la maîtresse du truand qui traverse la filmographie de Melville. Interrogé en 1961 au sujet des femmes dans son œuvre $^{12}$, le réalisateur reconnaissait une filiation directe entre ses héroönes et le modèle que représente l'ingénue fatale interprétée par Marilyn Monroe dans The Asphalt Jungle (John Huston, 1950) : une jeune femme belle et perdue à laquelle le cinéaste prête assez peu d'attention jusqu'à ce que, le plus souvent, elle finisse par trahir son amant par faiblesse ou par confort. On pourrait citer chez Melville Anne (Isabelle Corey) dans Bob le flambeur, Gloria (Monique Hennessy) dans Deux hommes dans Manhattan, Thérèse (Monique Hennessy à nouveau) dans Le Doulos ou encore Jane Lagrange (Nathalie Delon) dans Le Samouraï. Si ce type particulier de la femme fatale se retrouve dans les années 1960 jusque dans les films des anciens jeunes loups de la Nouvelle vague (par exemple La Sirène du Mississippi de François Truffaut en 1969 avec, déjà, Catherine Deneuve), Denitza Bantcheva et Roberto Chiesi ont bien montré dans leur étude historique du cinéma noir français à quel point ce motif faisait déjà à l'époque «davantage l'effet d'une survivance contre nature qu'une image crédible de l'éternel féminin ${ }^{13}$. ». Force est de constater le rôle balisé, presque caricatural qu'elles jouent chez Melville. Il apparaît particulièrement révélateur de s'intéresser à la façon dont elles sont poussées à la trahison. Le plus souvent, cela passe par leur humiliation physique ou verbale orchestrée par un homme (violence et torture de la part de Silien dans Le Doulos, discours paternaliste et infantilisant du commissaire dans Le Samourai). Mais, le plus souvent, l'instant précis de la dénonciation se trouve escamoté dans une 
ellipse dont l'intérêt dramatique - pour maintenir le suspense - se double d'une vision tranchée de la place des femmes dans le récit. La supposée trahison de Cathy dans Un flic prolonge d'une façon singulière ces ellipses récurrentes chez le cinéaste qui privent la traitresse de la parole. Simon téléphone à Cathy et lui demande de venir le chercher pour transporter une mallette de cocaïne. Alors qu'elle décroche le combiné, les équipes de Coleman tentent de tracer l'appel en parallèle. La conversation entre Simon et Cathy se termine par un silence de la femme lourd de sous-entendu: fait-elle volontairement durer leur échange pour laisser le temps aux policiers de localiser son amant? Comme pour le personnage de Mathilde dans L'Armée des ombres dont on ne peut que présumer qu'elle a fini par livrer tous les hommes du réseau, comme pour Jane Lagrange dont on se demande jusqu'au bout si elle n'a pas fini par dénoncer Jef Costello, la trahison de Cathy reste tue. L'attitude de Melville est alors double : dans un geste de cinéaste violent, il coupe la parole aux femmes (littéralement, comme on dit que l'on « coupe » une scène), mais dans le même temps il semble vouloir épargner leur honneur et refuser de faire peser sur elle la véritable culpabilité. Cette idée rejoint l'analyse souvent faite des films de gangsters du réalisateur à l'aune des principes de la tragédie classique ${ }^{14}:$ la faute ne pèse pas tant sur un personnage en particulier que sur le destin qui le manipule pour s'accomplir.

\section{La meurtrière}

5 La scène fondatrice de Cathy où elle tue un des hommes de Simon sur son lit d'hôpital de peur qu'il ne les compromette tous, confère au personnage une aura particulière. Il s'agit d'un exemple presque unique de femme meurtrière chez Melville, aucun autre homicide féminin n'étant montré, dans sa filmographie, avec un tel luxe de détails. Cathy se glisse furtivement dans la clinique habillée en infirmière et gravit l'escalier jusqu'à la chambre du blessé. Elle s'approche lentement du lit pour accomplir son forfait en insérant une seringue dans la transfusion et s'attarde un instant après que l'électrocardiogramme est devenu plat. On retrouve dans le début de la scène le motif du double attaché à Cathy qui ne se reflète plus dans un miroir comme dans la scène du baiser avec Coleman, mais dont l'ombre portée très marquée la précède et l'accompagne dans sa marche vers le lit funeste. À l'inverse des exécutions rapides perpétrées dans le film par les hommes en quelques coups de revolver, l'assassinat devient ici un processus technique lent dont Melville détaille chaque étape par un enchaînement de gros plans quasi-documentaires qui alternent avec des zooms à l'effet dramatique. L'autre grande différence avec les exécutions froides de western qui demeurent l'apanage des figures masculines réside dans les émotions que l'on peut lire sur le visage de Cathy. Dans un contexte ou l'underplay est érigé en règle d'or et où les hommes demeurent toujours impassibles, la respiration lourde de la femme à son entrée dans la chambre, puis la lèvre qu'elle mordille une fois le crime accompli, apparaissent presque comme des signes de jeu outranciers.

6 Il convient également d'analyser la technique du meurtre qui relève d'une perversion du rôle de soignante classiquement attribué à la femme, le domaine de la santé se trouvant chez Melville principalement pris en charge par des personnages féminins maternels. Il existe une première version de cette scène dans L'Armée des ombres, dont Denitza Bantcheva a souligné la parenté avec Un flic ${ }^{15}$. Dans le film de 1969, Mathilde vêtue en infirmière et deux hommes du réseau de Résistance déguisés en brancardiers s'introduisent dans un repère de la Gestapo pour tenter de sauver Félix, un des leurs 
fait prisonnier, afin, là aussi, de l'empêcher de parler. Dans ces deux situations en tous points identiques (par le nombre de personnages, la répartition des rôles, le travestissement, les obstacles, etc.), les moyens diffèrent (la libération ou le meurtre), mais le but à atteindre reste le même : préserver à tout prix la sécurité du groupe. Cette mission de protection d'un clan assimilé à une famille incombe à la femme dans les deux cas. Il existe ainsi une autre dimension du personnage de Cathy qui dépasse son statut de femme fatale standard et la rapproche non seulement de Mathilde, la mère par excellence du cinéma de Melville (qui sera d'ailleurs perdue par son tiraillement entre sa famille biologique et sa fidélité au groupe), mais aussi de Manouche dans Le Deuxième souffle dont le rôle auprès du Gu reste toujours ambigu, à la fois sœur protectrice et maîtresse. Cathy navigue de cette façon entre les deux seuls modes d'existence des femmes dans les films de gangsters melvilliens, maternelles ou fatales.

\section{«Transformer les hommes en femmes »"16}

\section{Blond(e)s ou brun(e)s}

7 Dans Un flic, Cathy appartient à un ensemble de personnages immédiatement identifiable, celui des blonds, qui partagent tous une forme de féminité. Elle apparaît de façon évidente chez la prostituée assassinée, tout comme chez Gaby l'indic transsexuelle, ou chez le jeune éphèbe, voire chez «La Valise ", le truand à l'aspect presque grotesque que l'on devine être un client de Gaby. Tous se trouvent liés par leur attirance sexuelle manifeste pour les hommes qui va de pair avec un certain caractère passif-avec tous les sous-entendus que ce terme comporte-car ils sont principalement mus par les autres, c'est-à-dire par les hommes bruns (Coleman et Simon) qui détiennent la force et orientent l'action. Pourtant, les rapports entre Gaby et le commissaire nous permettent de remettre en cause le modèle viriliste que Melville semble vouloir imposer. En témoigne leur première scène commune dans laquelle Coleman gare sa voiture en attendant Gaby, qui arrive à son tour, se range et vient en courant à sa rencontre pour le rejoindre sur le siège passager. Un trouble naît du constat que l'on identifie immédiatement Gaby à un personnage transsexuel, alors même qu'elle est interprétée par une femme (Valérie Wilson), en raison de l'outrance de son maquillage, de son timbre de voix grave et de ses manières féminines exagérées, comme si le trop-plein de féminité de Gaby venait, en miroir, jeter le doute sur la masculinité excessive de Coleman (fig. 3). De plus, cette scène est très exactement tournée comme une rencontre amoureuse avec l'arrivée de la Jaguar de l'indic sur une petite musique de Noël jouée en hors-champ, le travelling qui suit sa course, les visages en champ-contrechamp filmés en plans rapprochés (exactement comme dans la première scène entre Coleman et (athy), et la musique de cordes qui arrive à la fin. On note également que le commissaire, en regardant Gaby, esquisse l'un des deux seuls sourires qu'il consent dans le film - l'autre apparaissant, de façon particulièrement significative, un peu plus tard lors de sa première scène avec Cathy, quand il la regarde depuis son piano. Gaby et Cathy incarnent ainsi les deux faces du désir amoureux de Coleman dans un triangle que Melville a déjà expérimenté dans Le Samouraï en 1967, selon un dispositif légèrement différent. En effet, Alain Delon y interprétait Jef Costello, double hors-la-loi du commissaire de 1972, pris entre deux femmes, l'une blanche (Jane Lagrange) et l'autre noire (Valérie). La question raciale jouait alors le même rôle que la problématique homosexuelle dans Un flic. Il y a d'un côté une femme blonde, en 
apparence innocente et dévouée, mais sur laquelle plane le spectre de la traîtrise, et, de l'autre, un personnage clandestin, inacceptable, mais inconditionnellement bon. D'une certaine façon, Melville prend en charge une dimension morale inattendue en corrigeant les injustices tragiques du récit : si Valérie et Gaby subissent les foudres des autres protagonistes, jamais le spectateur ne les soupçonne d'avoir trahi. Elles incarnent deux innocentes martyres.

Fig. 3

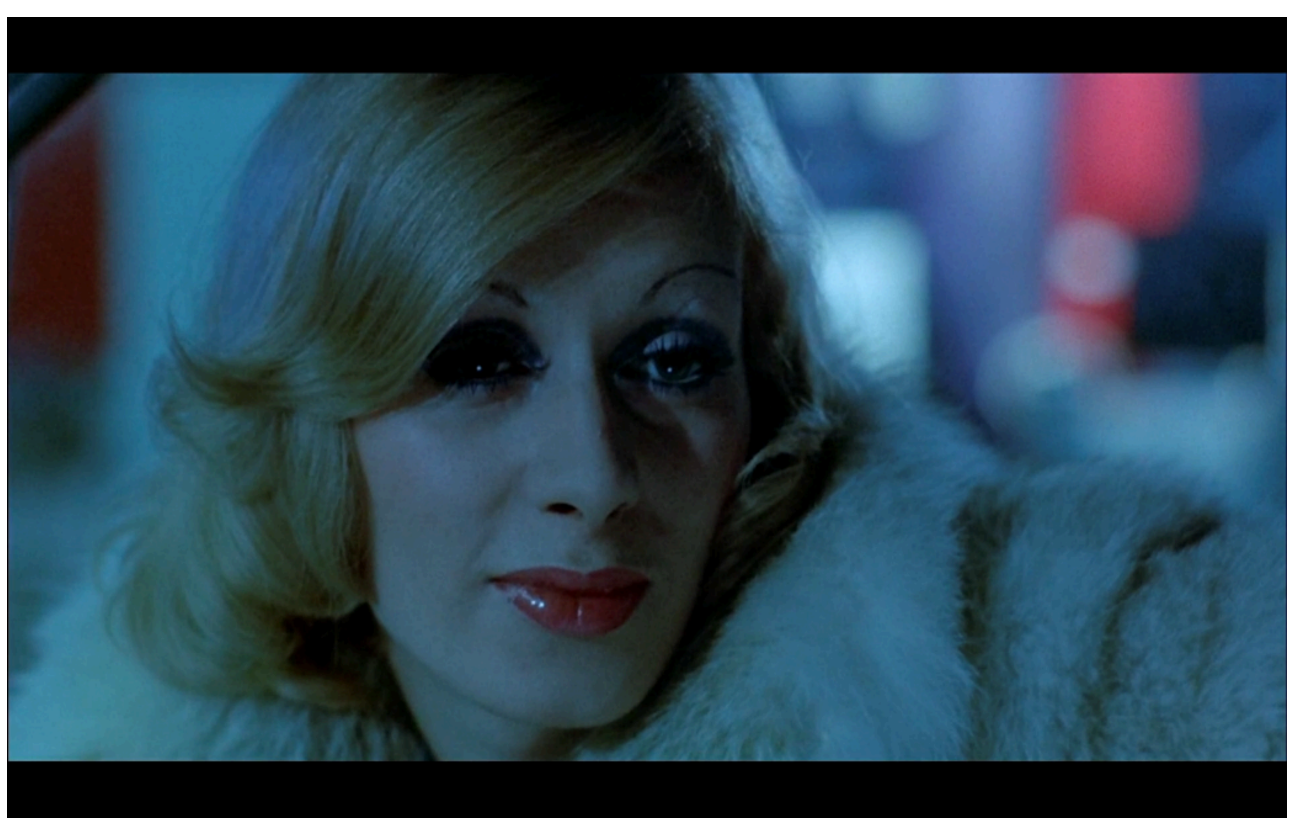

Un flic, Jean-Pierre Melville, 1972

(c) Sophie Dulac Distribution

\section{Sexe déviant et violence virile}

8 Melville n'a jamais fait mystère du sous-texte homosexuel de nombre de ses films. Il l'a déjà explicité dans L'Aîné des Ferchaux en 1963 à propos duquel il expliquait que Charles Vanel tombait, pour la première fois, amoureux de Jean-Paul Belmondo. Dans une rencontre avec des spectateurs à l'occasion de la sortie du film, il ajoutait : «J'ai connu des hommes extrêmement virils qui, tout à coup, ont subi ce genre de choses ${ }^{17}$ ». La virilité n'exclut pas, dans son cinéma, le désir homosexuel, mais celui-ci demeure, pour les bruns (contrairement aux blonds pour qui il s'affiche de manière évidente), systématiquement de l'ordre du refoulé et de la violence. La plupart de ses intrigues qualifiées de «films d'hommes " reposent sur un couple masculin équivoque : on peut penser à la relation Corey/Vogel dans Le Cercle rouge ou Silien/Faugel dans Le Doulos pour ne citer que les exemples les plus manifestes. La dernière scène entre Coleman et Gaby est, à cet égard, édifiante. Gaby est conduite dans le bureau du flic parce que celuici la soupçonne de l'avoir volontairement mené sur une fausse piste. S'ensuit une scène d'humiliation particulièrement violente en forme de rupture sentimentale. Coleman dirige sa brutalité contre l'identité sexuelle de l'indic: avant même de lui expliquer pourquoi il l'a faite venir, il la frappe, la fait tomber à terre, et l'insulte sur le mode du masculin (" connard », « à partir de maintenant tu vas t'habiller en homme »), avant de 
lancer à ses subordonnés un «foutez-moi ça dehors » qui, par l'utilisation d'une forme grammaticale neutre et réservée aux choses, insiste sur l'indistinction de genre qui conduit ici à une forme de déshumanisation. Pourtant, d'un point de vue technique, cette scène est très proche de leur première rencontre. Le travelling qui accompagnait la course de Gaby vers Coleman est ici remplacé par un autre travelling filmant l'indic, cette fois de profil, quittant le commissariat en pleurs. La même musique revient à la fin des deux scènes et les échelles des plans, privilégiant les cadrages serrés sur les visages, se répondent.

9 Pour remonter la généalogie de cette ultime confrontation, on peut citer une séquence du Doulos entre Silien et Thérèse qui commence comme un flirt et se termine dans un mélange de violence et d'érotisme empruntant, de façon évidente, à l'iconographie sadomasochiste. Silien pénètre dans l'appartement de Thérèse, fait mine de la complimenter, puis la bat violemment jusqu'à ce qu'elle s'écroule. Inconsciente, elle est ligotée à l'aide d'une corde blanche et attachée au radiateur. Dans les entretiens qu'il a accordés, Melville lie lui-même presque systématiquement érotisme et violence. En 1968, il déclarait sur un ton qui résonne particulièrement avec ces scènes : « La violence représentée au cinéma et à la télévision est un besoin absolu chez l'homme. Au même titre qu'avant-guerre les maisons closes étaient tellement logiques. Les hommes ont besoin de compenser quelque chose dont ils sont privés. La violence fait partie de l'homme. La fermeture des maisons closes, il y a vingt-cinq ans, se traduit par cette débauche extraordinaire de publicité érotique, dont on parle beaucoup, par cette escalade démente dans la pornographie au cinéma ${ }^{18} »$. La violence et le sexe semblent représenter pour lui deux versants d'une même pulsion brutale. De là sans doute vient le fait que, dans ses films, actes violents et actes sexuels ne se différencient pas, qu'un pistolet brandi vaut pour un phallus en érection et qu'une séance de torture reprend des codes du bondage, non pas suivant les principes d'une métaphore éculée, mais dans une logique d'indistinction totale où les deux termes ne se contentent pas de représenter la même chose, mais sont une seule et même vérité du personnage.

\section{Fétichisme wildien et obsession hitchcockienne}

10 L'autre dimension érotique importante des personnages virils chez Melville vient de la fascination du cinéaste pour leur apparence qui confine, selon son propre terme, au « fétichisme ${ }^{19}$ ». Il a convoqué la référence à Oscar Wilde de façon surprenante dans une lettre ouverte au cinéaste états-unien Billy Wilder en 1970 à propos de son film The Private Life of Sherlock Holmes ${ }^{20}$. Après avoir reconnu les similitudes entre leurs deux œuvres: «Cela m'était d'autant plus agréable [Melville se réjouissait qu'un de ses collaborateurs travaille sur le film de Wilder] que, de mon côté, j'avais décidé Alain Delon à tourner avec moi, tout de suite après Le Cercle rouge, la Vie Privée d'Arsène Lupin, et que le parallèle de nos deux démarches, de notre rencontre presque, me troublait. ", le réalisateur français assimile les déboires subis par le film de Wilder aux persécutions dont fut victime Oscar Wilde : "Cette longue lettre, cher Billy, pour vous dire qu'ici nous avons compris qu'en 1890 il fallait beaucoup de courage à Holmes et à Watson pour vivre ensemble, dans le temps où Wilde écrivait une ballade et à vous en 1970 autant de courage et d'humour et de génie pour nous montrer enfin le vrai visage du premier private eye (détective privé) de l'histoire de la littérature. / Donc, pas de Motion Picture Award cette année, Billy. / Mais soyez attentif dans la lecture de votre courrier : une carte postale peut vous être adressée de la prison de Reading - symbole de toutes 
les prisons où l'on enferme une quelconque liberté - elle sera signée d'un prénom et c'est quand même un signe : "Oscar ». / P.-S. Wilder: traduction: plus de Wilde." Melville évoque ici l'ambiguïté homosexuelle qui parcourt le début du film de Wilder et qui, sur un mode comique, rappelle, il est vrai, les couples virils melvilliens. Mais le modèle de l'écrivain anglais s'ancre plus en profondeur dans les filmographies des deux cinéastes et on peut aisément en trouver les traces chez Melville, non seulement dans le dandysme extrême dont il fait preuve pour ses héros, mais également dans le rôle quasiment métaphysique que joue leurs reflets dans des plans de miroirs que l'on retrouve dans la majorité de ses films. Une scène d'Un flic représente le meilleur exemple de l'importance de l'apparence qui confine à une certaine coquetterie pour le protagoniste melvillien. Simon, débarqué dans un train depuis un hélicoptère, passe de longues minutes dans un petit cabinet de toilettes à se changer en apportant le plus grand soin à sa tenue, ainsi qu'à sa coiffure qu'il repeigne à plusieurs reprises (fig. 4). Antoine de Baecque résume cette obsession en se demandant «si la panoplie melvillienne du truand ne visait finalement qu'à cela : transformer l'homme en femme [...].» et il ajoute: «Car Melville met à filmer les hommes la sophistication et le glamour que les grands artistes hollywoodiens ont mis à sublimer les plus grandes stars féminines [...]. $»^{21}$ Il existe en effet dans la démarche du réalisateur une inversion des stéréotypes de genres mis en place par le cinéma états-unien. Dans Un flic, Catherine Deneuve porte presque toujours la même robe, créée par Yves Saint-Laurent dans le but de masquer sa grossesse, alors que toute la portée érotique et maniaque du vêtement élégant qui se défait progressivement se trouve prise en charge par les hommes. La longue scène de Simon qui s'habille dans les toilettes du train n'est autre chose qu'un effeuillage inversé, tandis que tout au long du film les cols de chemise s'ouvrent, les vestes se déboutonnent, les cheveux se décoiffent au gré des interrogatoires violents ou des face-à-face amoureux (on retrouve ici l'excitation équivalente entre sexe et brutalité masculine).

Fig. 4

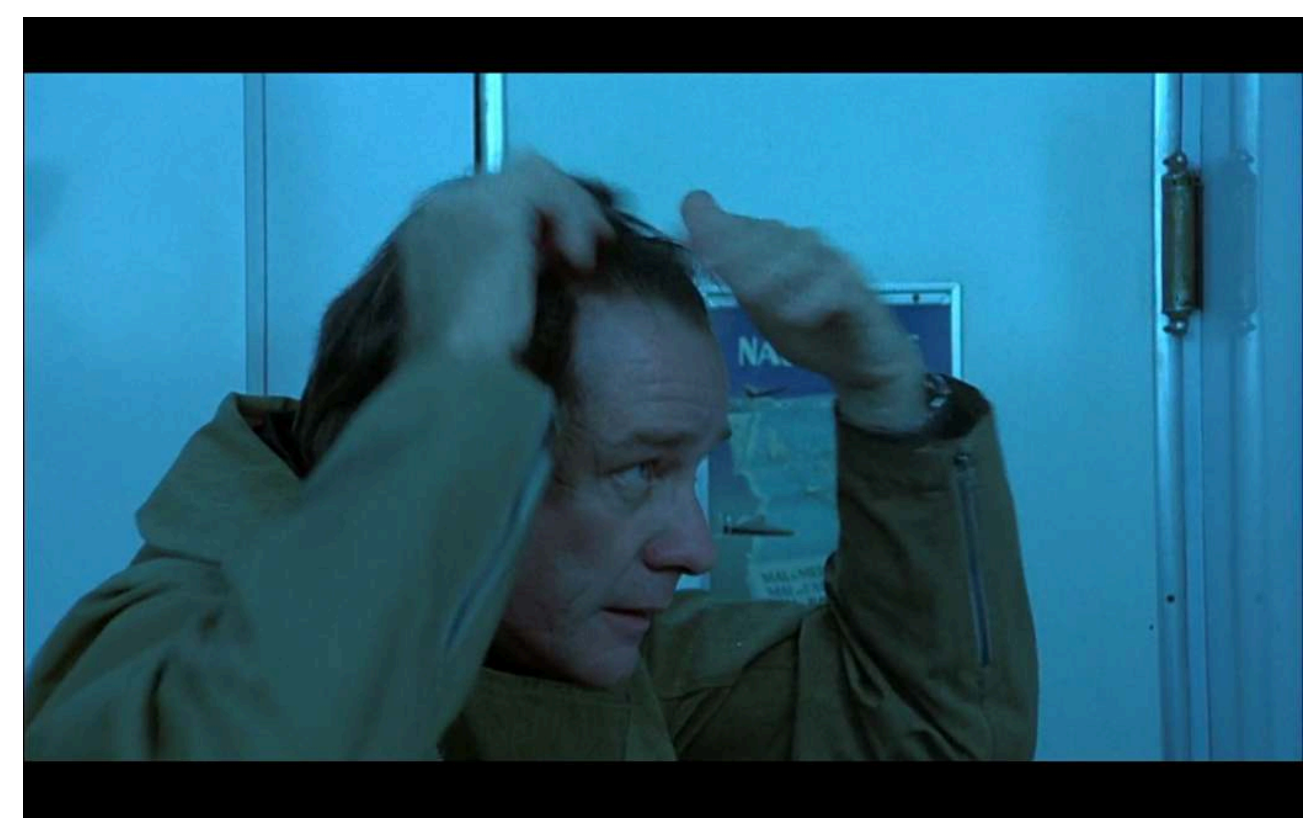

Un flic, Jean-Pierre Melville, 1972

(c) Sophie Dulac Distribution 
11 En cela, le miroir représente un fétiche important. Il est à la fois le lieu de l'admiration de soi (la glace du train devant laquelle Simon se repeigne) et une réplique du portrait de Dorian Gray dans laquelle le héros se voit offrir une occasion unique de se découvrir tel qu'il est, le plus souvent au moment de sa mort. La fin du Doulos où Silien s'effondre devant son reflet en reste la meilleure illustration. Le dernier plan d'Un flic n'est pas étranger à cette logique (fig. 5). Coleman et l'inspecteur Morand sont assis côte à côte dans la voiture du commissaire. Dans un plan fixe, la caméra s'attarde sur leurs deux visages antagonistes qui occupent chacun la moitié de l'écran : à gauche le trouble de Morand et à droite, en miroir, l'impassibilité de Coleman. Après une dernière coupe, le regard vide du commissaire emplit l'écran sur lequel vient s'inscrire le générique.

Fig. 5

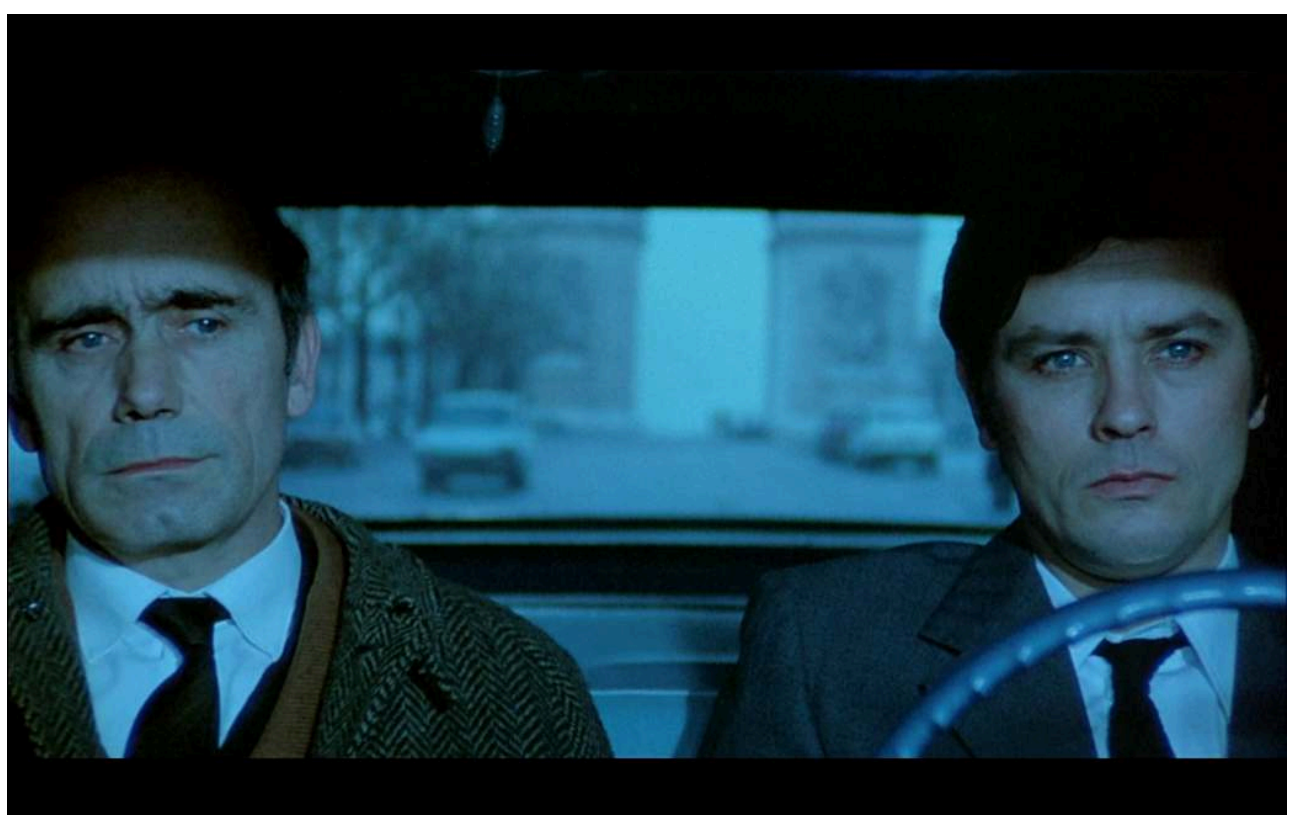

Un flic, Jean-Pierre Melville, 1972

(C) Sophie Dulac Distribution

Si l'on reprend les réflexions d'Antoine de Baecque à propos de l'obsession melvillienne pour les hommes et de la fascination hollywoodienne pour les femmes, une citation de Melville concernant les stars se révèle particulièrement troublante : «Je l'ai habillée, je me suis promené des jours et des jours à Paris avec elle à la recherche d'un manteau, d'un chapeau, d'une cravate, d'un petit détail vestimentaire, des lunettes, j'ai été essayer les fausses moustaches chez Pontet avec elle. [...] Je dis elle parce que c'est la star, mais c'est en général un homme ${ }^{22} »$. Il est difficile de ne pas lire ces phrases sans y voir une description presque mot à mot de la séquence de Vertigo d'Alfred Hitchcock (1958) dans laquelle Scottie tente de recréer avec Judy l'image de la défunte Madeleine en faisant avec elle le tour des boutiques de San Francisco pour choisir ses vêtements. Ce passage étant lui-même révélateur d'une certaine pathologie hitchcockienne, il semble possible d'établir un lien entre le rapport fétichiste du cinéma d'Hitchcock à la femme et une tendance identique dirigée vers l'homme chez Melville. Les deux filmographies représenteraient ainsi une obsession identique et partageraient la même origine fantasmatique. Pour poursuivre cette comparaison, avançons qu'un champ de réflexion s'ouvre autour des sorts contraires réservés aux personnages féminins et 
masculins chez Hitchcock et chez Melville. Dans les films de ce dernier, les femmes, même violentées ou assassinées ne saignent pas, à la différence des héroïnes hitchcockiennes. Même si le réalisateur français n'est pas un grand sanguinaire, le sang des hommes coule parfois et de manière hautement symbolique, à l'image du filet qui sort de la bouche de Jef Costello à la fin du Samouraï. Le héros peut également se trouver complètement défigurés, comme Félix et Jean-François dans L'Armée des ombres, alors que le corps féminin est épargné jusque dans les pires circonstances (dans Un flic le cadavre de la prostituée reste étrangement immaculé). Une étude intéressante reste à mener sur la représentation du sang des femmes chez Hitchcock comme une souillure (la scène de la douche de Psycho où il est délibérément identifié au sang menstruel) et comme la marque d'un péché (l'assimilation de Melanie Daniels à une sorcière dans The Birds). Il pourrait être appréhendé comme l'équivalent de la destruction du corps masculin chez Melville, presque systématiquement tué à la fin.

\section{Conclusion}

Pour conclure, revenons sur une citation de Melville dans laquelle il tente de justifier la relative absence de femmes dans ses films : « Un cinéma sans femme car, je m'en excuse auprès des dames (que j'aime beaucoup), l'homme ne peut se sublimer qu'en dehors de ses désirs, de sa libido et de ses complexes ${ }^{23}$.» Ces derniers termes définissent précisément ce avec quoi se débat Coleman dans Un flic. Faut-il alors comprendre le film comme la quête désespérée d'absolu d'un homme en proie à ces trois démons, incarnés par des personnages troubles de traîtresses ou de tentatrices? Il semble toutefois réducteur d'affirmer que le cinéma de Melville est "sans femme». Si certaines des héroïnes que nous avons citées au cours de cette article relèvent en apparence de stéréotypes de genres éculés ${ }^{24}$, elles dissimulent d'autres grandes figures féminines dans l'œuvre du réalisateur, au-delà de ses seuls films de gangsters. Si l'on se penche sur Léon Morin, prêtre (1961), on constate que tout le récit consiste, comme pour Un flic, à confronter Barny (Emmanuelle Riva) à ses désirs, sa libido et ses complexes, sous l'apparence non seulement de Léon Morin, mais aussi de Sabine Levy, sa patronne dont elle est amoureuse ${ }^{25}$. À l'origine, cette première partie homosexuelle devait être plus développée, le prêtre n'arrivant qu'au deuxième tiers de l'histoire ${ }^{26}$. Barny, comme Thérèse (Juliette Gréco) dans Quand tu liras cette lettre (1953), comme la jeune fille incarnée par Nicole Stéphane dans Le Silence de la mer (1949), ou, dans une certaine mesure, le couple de lesbiennes dans Deux hommes dans Manhattan (1959), représente le pendant féminin des flics et des voyous qui cherchent à se sublimer mais restent cloués au sol par leurs pulsions inavouables et par la force du destin.

\section{NOTES}

1. Voir notamment: Raymond Lefevre, "Journal d'un animateur de journées très animées ", Cinéma 63, nº 81, décembre 1963, pp. 76-80 
2. Christian Ledieu, «Études. Jean-Pierre Melville», Études cinématographiques, $\mathrm{n}^{\text {os }} 6-7,4^{\mathrm{e}}$ trimestre 1960, p. 443. Cet article est par ailleurs l'un des premiers véritablement intéressants que nous ayons retrouvés.

3. Henry Chapier, «Un film de la main gauche », rubrique "Le film du jour », Combat, 28 octobre 1972.

4. Claude Mauriac, L'Express, 30 octobre 1972.

5. A. C., « Un Flic », Les Échos, 27 octobre 1972.

6. Edmond Gilles, « Un bon film de plus... . "Un flic”, de J.-P. Melville », L'Humanité, 15 novembre 1972.

7. Jean-Louis Tallenay, « Triste servitude du métier », Le Monde, 31 octobre 1972.

8. Idem.

9. Edmond Gilles, op. cit. note 6 .

10. Claude Beylie, Bertrand Tavernier, «Entretien avec Jean-Pierre Melville », Cahiers du cinéma, $\mathrm{n}^{\circ} 124$, octobre 1961, p. 14.

11. Au sujet du masque et du déguisement, lire : Claude Beylie, « Entretien avec JeanPierre Melville », Cinéma 69, nº 140, novembre 1969, pp. 125-126.

12. Claude Beylie, Bertrand Tavernier, art. cité, p. 13.

13. Denitza Bantcheva, Roberto Chiesi, Le film noir français, Rome, Gremese, 2015, pp. 39-40.

14. Voir notamment: Alain Garel, «Melville, Jean-Pierre - (1917-1973). Le film noir comme tragédie moderne", Encyclopcedia Universalis [en ligne], consulté le 16 septembre 2019. URL: http://www.universalis.fr/encyclopedie/jean-pierre-melville/

15. Denitza Bantcheva, Jean-Pierre Melville de l'œeuvre à l'homme, Paris, Éditions du Revif, 2007, p. 99.

16. Antoine de Baecque, Jean-Pierre Melville, une vie, Paris, Seuil, 2017, p. 153.

17. Raymond Lefevre, art. cité note 1, p. 80 .

18. Patrick Bureau, «Chroniques melvilliennes » [2 ${ }^{\text {de }}$ partie], Cinéma $68, n^{\circ} 129$, octobre 1968 , p. 87-88.

19. Idem, p. 79-80.

20. Jean-Pierre Melville, L'Aurore, décembre 1970, repris dans Positif, $n^{\circ} 679$, septembre 2017, p. 115.

21. Antoine de Baecque, op. cit. note 16.

22. P. Bureau, art. cité note 18, p. 76 .

23. Jean-Pierre Melville, "Hommage à Jacques Becker », Cahiers du cinéma, nº 107, mai 1960. Cité dans A. de Baecque, op. cit. note 16.

24. Fabien Baumann, «La poule melvillienne, ce vide désirable », Positif, $n^{\circ} 679$, septembre 2017, p. 108-110.

25. À ce sujet, lire : Rui Nogueira, Le cinéma selon Jean-Pierre Melville, Paris, Éditions de l'Étoile/

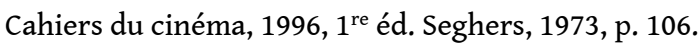

26. Idem.

\section{RÉSUMÉS}

Si dans la littérature les œuvres de la seconde moitié de la carrière de Jean-Pierre Melville sont souvent qualifiées de "films d'hommes ", Un flic (1972), sa dernière réalisation, est celle qui 
interroge le plus ouvertement la question du genre. L'intrigue policière y devient le prétexte pour présenter une galerie de personnages aux identités et assignations sexuelles troubles. Ainsi, la dichotomie entre le personnage de Cathy, femme fatale et meurtrière en blouse blanche, et celui de Gaby, indic transsexuelle qui subit l'humiliation et les coups répond au parallèle entre la figure du flic et celle du truand. Cette étude détaillée des mécanismes de la violence qui fondent le film permet d'interroger son ambiguïté, tout en l'inscrivant dans la morale genrée qui parcourt toute la filmographie du cinéaste.

Although the movies of the second half of Jean-Pierre Melville's career are often referred to in the literature as "men's films", Un flic (1972), his last work, is the one that most openly questions the issue of gender. The detective story becomes the pretext to present a gallery of characters with murky sexual identities and attributions. Thus, the dichotomy between the character of Cathy, a femme fatale and a murderer in a lab coat, and that of Gaby, a transsexual police informer made to suffer humiliation and beatings, matches the parallel between the cop and the mobster. This detailed study of the mechanisms of violence that underlie the film questions its ambiguity, while also ascribing it as an element of the gender-based morality that runs through the director's filmography.

\section{INDEX}

Mots-clés : Cinéma, Jean-Pierre Melville, femme fatale, genre, homosexualité, transsexualité, film noir, Alfred Hitchcock, Catherine Deneuve, Alain Delon

Keywords : Cinema, Jean-Pierre Melville, femme fatale, gender, homosexuality, transsexuality, film noir, Alfred Hitchcock, Catherine Deneuve, Alain Delon

\section{AUTEUR}

\section{THOMAS LEQUEU}

Doctorant à l'École du Louvre sous la direction de François-René Martin, Thomas Lequeu est également critique de cinéma pour la revue Critikat. Il a par ailleurs travaillé sur l'œuvre du peintre George Desvallières, dont il est co-auteur du catalogue raisonné. En 2020, il a rejoint l'équipe de la villa Noailles en qualité de commissaire d'exposition et archiviste.

Thomas Lequeu is a post-graduate student at the École du Louvre, where he wrote his thesis under the direction of François-René Martin. His research focuses on the representations of fear in film during the Cold War. He is also a film critic for the review Critikat. He has also studied the work of painter George Desvallières, of whose catalogue raisonné he is a co-author. In 2020, he became the curator and archivist at Villa Noailles. 